\title{
EXTENSION ON THE FUZZY INTEGRAL BASED ON $\oplus$-DECOMPOSABLE MEASURE
}

\author{
MAMADOU TRAORE
}

(Received 13 April 2001)

\begin{abstract}
We extend the concept of fuzzy integral based on $\oplus$-decomposable measure from nonnegative fuzzy measurable function to extended real-valued fuzzy measurable function. Further investigations of fuzzy integrals based on pseudo-additive decomposable measure are carried out. Meanwhile, the space $(S(\mu), \sigma(\cdot, \cdot))$ of all fuzzy measurable function will be proved to be a pseudo-metric space. Finally, as an application of this extension the Pettis integral will be obtained for that kind of fuzzy integral.
\end{abstract}

2000 Mathematics Subject Classification. 03B52.

1. Introduction. The theory of fuzzy integral is an important part of fuzzy analysis. The concept of fuzzy sets suggested by Zadeh [10] is a mathematical expression of sets without precise boundaries and has been applied to various problems in engineering.

Fuzzy measures have been intensively discussed since Sugeno [7] defined a fuzzy measure as a measure having monotonicity instead of additivity. Weber [9] proposed $\perp$-decomposable measures where the additivity of measure is weakened. Special type of decomposable measure and integral for nonnegative measurable function have been done by many mathematicians (see [2, 3, 5, 8, 9] etc.). The pseudo-additive measure and integral investigated by Sugeno and Murofoshi [8] are special case of $\perp$-decomposable measure and integral. In [4], the author gave some convergence theorem for fuzzy integral based on $\oplus$-decomposable measure. It is natural to ask whether we can define the fuzzy integral for extended real-valued fuzzy measurable function. The answer is just the purpose of this paper. Since the fuzzy integral based on $\oplus$-decomposable measure will be defined by using the classical Lebesgue integral, we will use some results of classical measure theory. The paper deals at first with the definition and basic properties of fuzzy integral based on $\oplus$-decomposable measure for extended real-valued fuzzy measurable function. Next, Fisher theorem will be obtained for this kind of fuzzy measure, and the absolute integrability will be investigated. After that the space $(S(\mu), \sigma(\cdot, \cdot))$ of all $\mu$-measurable function will be proved to be a pseudo-metric space. Finally, as an application of this extension the Pettis integral will be obtained for that kind of fuzzy integral.

2. Preliminaries and pseudo-additive integral of extended real-valued measurable function. Let $[a, b]$ be a closed subinterval of $[-\infty, \infty]$ (in some cases we will also take semiclosed subinterval). The full order on $[a, b]$ will be denoted by $\leq$, this is the usual order of the real line but it can also be another order. 
DEFINITION 2.1 (see [2]). Let $[a, b]$ be a closed real interval and $\oplus:[a, b] \times[a, b] \rightarrow$ $[a, b]$ be a two place function satisfying the following conditions:

(1) $\oplus$ is commutative,

(2) $\oplus$ is nondecreasing in each place,

(3) $\oplus$ is associative,

(4) $\oplus$ has either $a$ or $b$ zero element, that is, either $\oplus(a, x)=x$ or $\oplus(b, x)=x$ is called a pseudo-addition.

Definition 2.2 (see [2]). A pseudo-multiplication $\otimes$ is a two place function $\otimes$ : $[a, b] \times[a, b] \rightarrow[a, b]$ satisfying the following conditions:

$(1) \otimes$ is commutative,

$(2) \otimes$ is nondecreasing in each place,

(3) $\otimes$ is associative,

(4) there exists a unit element $e \in[a, b]$ such that $\otimes(x, e)=x$ for all $x \in[a, b]$.

Throughout, $\oplus$ is assumed to be an Archimedean pseudo-addition and $([a, b], \oplus, \otimes)$ is assumed to be a semi-ring with pseudo-operation defined by monotone and continuous generator $g$. In this case we will consider only strict pseudo-addition, such that the function $\otimes$ is continuous and strictly increasing in $(a, b) \times(a, b)$. By Aczel's representation we have that, for each strict addition $\oplus$ there exists a monotone function $g ; g:[0, b] \rightarrow[0, \infty]$ such that

$$
g(0)=0, \quad u \oplus v=g^{-1}(g(u)+g(v)) .
$$

Using a generator $g$ of strict pseudo-addition $\oplus$, we can define pseudo-multiplication $\otimes$ :

$$
u \otimes v=g^{-1}(g(u) \times g(v)) .
$$

We will notice that this is the only way to define a pseudo-multiplication $\otimes$ which is distributive with respect to $\oplus$ and generated by the function $g$.

Definition 2.3 (see [6]). A set function $\mu: \Omega \rightarrow[a, b]_{+}$is a $\oplus$-decomposable measure if the following hold:

$$
\mu(\varnothing)=0, \quad \forall A, B \in \Omega, \quad A \cap B=\varnothing \quad \text { then } \quad \mu(A \cup B)=\mu(A) \oplus \mu(B) .
$$

A $\oplus$-decomposable measure is said to be $\sigma$ - $\oplus$-decomposable if for all $\left(A_{n}\right)_{n \in \mathbb{N}} \subset \Omega$, $A_{i} \cap A_{j}=\varnothing, i \neq j$, we have

$$
\mu\left(\bigcup_{n=1}^{\infty} A_{i}\right)=\oplus_{i=1}^{\infty} \mu\left(A_{i}\right)
$$

with

$$
\oplus_{i=1}^{\infty} \mu\left(A_{i}\right)=\lim _{n \rightarrow \infty} \oplus_{i=1}^{n} \mu\left(A_{i}\right)
$$

DEFINITION 2.4 (see [2]). Let $(X, \Omega, \mu)$ be a $\oplus$-measure space and consider a simple measurable function $f$ such that $f(w)=\sum_{k=1}^{n} a_{k} \cdot I_{A_{k}}(w)$ where $A_{k} \in \Omega, k=$ 
$1,2,3, \ldots, n$ is a partition of $\Omega$. Then the fuzzy integral based on pseudo-additive measure is defined by

$$
\int_{X} f \otimes \mu=\oplus_{k=1}^{n}\left(a_{k} \otimes \mu\left(A_{k}\right)\right) .
$$

The above fuzzy integral is well defined.

Proposition 2.5 (see [1]). Let $f$ be a measurable positive function, then there exists a sequence $\left(\varphi_{n}\right)$ of increasing simple positive function such that for all $x \in X, f(x)=$ $\lim _{n \rightarrow \infty} \varphi_{n}(x)$.

DEFINITION 2.6. Let $f$ be a measurable function, $\left(\varphi_{n}\right)$ is a sequence of simple functions, $\varphi_{n} \rightarrow f$, $\mu$ be a $\sigma$ - $\oplus$-decomposable measure. Then

$$
\int_{X} f \otimes \mu=\lim _{n \rightarrow \infty} \int_{X} \varphi_{n} \otimes \mu .
$$

TheOrem 2.7 (see [4]). Let $(X, \Omega, \mu)$ be a $\oplus$-measure space, $f$ a nonnegative measurable function. And let

$$
\begin{aligned}
& a \oplus b=g^{-1}(g(a)+g(b)), \\
& a \otimes b=g^{-1}(g(a) \cdot g(b)) \quad \text { for } a, b \in \mathbb{R} .
\end{aligned}
$$

Then (2.6) and (2.7) can be defined by

$$
\int_{X} f \otimes \mu=g^{-1}\left[\int_{X}(g \circ f) d(g \circ \mu)\right]
$$

for any simple or measurable function, where $g \circ \mu=\gamma$ is an ordinary measure and $\int_{X} d(g \circ \mu)$ is the Lebesgue integral.

Proof. See [4].

REMARK 2.8. We say that $f$ is pseudo-integrable if for all $A \in \sum, \int_{A} f \otimes \mu<\infty$.

Definition 2.9. Let $(X, \Omega, \mu)$ be a fuzzy measure space, $f: X \rightarrow[-\infty, \infty] \mu$ measurable, and

$$
f^{+}(t)=\left\{\begin{array}{ll}
f(t), & f(t) \geq 0, \\
0, & f(t)<0,
\end{array} \quad f^{-}(t)=\left\{\begin{array}{ll}
0, & f(t)>0, \\
-f(t), & f(t) \leq 0,
\end{array} \quad(t \in X) .\right.\right.
$$

Clearly, $f^{+}$and $f^{-}$are also $\mu$-measurable and $f(t)=f^{+}(t)-f^{-}(t)(t \in \Omega)$.

If $f^{+}$and $f^{-}$are also pseudo-integrable on $A$, then we say that $f$ is pseudo-integrable on $A$ and define

$$
\int_{A} f(t) \otimes \mu=\int_{A} f^{+}(t) \otimes \mu-\int_{A} f^{-}(t) \otimes \mu .
$$

In the sequel we denote

$$
\begin{aligned}
S(\mu) & =\{f: X \rightarrow[-\infty, \infty]: f \text { is } \mu \text {-measurable and finite a.e. on } X\}, \\
L^{1}(\mu) & =\{f: X \rightarrow[-\infty, \infty]: f \text { is pseudo-integrable }\} .
\end{aligned}
$$


Proposition 2.10. Let $(X, \Omega, \mu)$ be a finite pseudo-additive measure space, then the following hold:

(1) If for all $A \in \Omega, \mu(A)=0$ then $\int_{A} f \otimes \mu=0$.

(2) $\int_{A} f \otimes \mu=\int_{X} f \cdot X_{A} \otimes \mu$.

(3) If $f \in L^{1}(\mu)$, then $-f \in L^{1}(\mu)$ and $\int_{A}(-f(t)) \otimes \mu=-\int_{A} f(t) \otimes \mu$.

(4) $f \in L^{1}(\mu), c \in(-\infty, \infty)$, then $c f \in L^{1}(\mu)$, and

$$
\int_{A} c \otimes \mu= \begin{cases}c \otimes \mu(A) & \text { if } c \geq 0, \\ -(-c \otimes \mu(A)) & \text { if } c<0 .\end{cases}
$$

Proof. (1) and (2) are obvious.

(3) We have that $(-f)^{+}(t)=f^{-}(t),(-f)^{-}(t)=f^{+}(t)(t \in \Omega)$, so by Definition 2.9 we have $-f \in L^{1}(\mu)$ and

$$
\begin{aligned}
\int_{A}(-f(t)) \otimes \mu & =\int_{A}(-f)^{+}(t) \otimes \mu-\int_{A}(-f)^{-}(t) \otimes \mu \\
& =\int_{A} f^{-}(t) \otimes \mu-\int_{A} f^{+}(t) \otimes \mu \\
& =-\left(\int_{A} f^{+}(t) \otimes \mu-\int_{A} f^{-}(t) \otimes \mu\right) \\
& =-\int_{A} f(t) \otimes \mu .
\end{aligned}
$$

(4) By (3) and similarly for pseudo-additive integral for nonnegative measurable function we conclude the proof.

Proposition 2.11. Let $\mu$ be a pseudo-additive measure and $f$ an extended realvalued measurable function; $f$ is pseudo-integrable if and only if $|f|$ is pseudointegrable and

$$
\left|\int_{X} f \otimes \mu\right| \leq\left\{\begin{array}{l}
\int_{X} f^{+} \otimes \mu \\
\int_{X} f^{-} \otimes \mu
\end{array} \leq \int_{X}|f| \otimes \mu .\right.
$$

\section{PROOF}

NECESSITY. Suppose that $f$ is pseudo-integrable then $\int_{X} f^{+} \otimes \mu<\infty$ and $\int_{X} f^{-} \otimes \mu<$ $\infty$, but $|f|=f^{+}+f^{-}$that implies

$$
\int_{X}|f| \otimes \mu=\int_{X} f^{+} \otimes \mu+\int_{X} f^{-} \otimes \mu=g^{-1}\left(\int_{X} f^{+} \otimes \mu+\int_{X} f^{-} \otimes \mu\right)<\infty .
$$

Thus $|f|$ is pseudo-integrable.

SUFFICIENCY. Suppose that $|f|$ is pseudo-integrable then $\int_{\Omega}|f| \otimes<\infty$ but $\int_{\Omega} f^{+} \otimes$ $\mu \leq \int_{\Omega}|f| \otimes<\infty$ and $\int_{\Omega} f^{-} \otimes \mu \leq \int_{\Omega}|f| \otimes<\infty$ thus $\int_{\Omega} f^{+} \otimes \mu$ and $\int_{\Omega} f^{-} \otimes \mu$ are two finite quantities, so according to Definition $2.9, f$ is pseudo-integrable.

THEOREM 2.12 (Fisher theorem). Let $(X, \Omega, \mu)$ be a pseudo-additive measure space. For every measurable sequence $\left(f_{n}\right)_{n \in \mathbb{N}}$ which converge in measure to $f$, we can extract a subsequence $\left(f_{n_{k}}\right)_{k \in \mathbb{N}}$ which converges to $f \mu$ a.e. 
Proof. By hypothesis $\left(f_{n}\right)$ converges in measure to $f$, so

$$
\begin{aligned}
\forall \varepsilon>0 ; \quad \lim _{n \rightarrow \infty} \mu & \left(\left\{t \in X:\left|f_{n}(t)-f(t)\right| \geq \varepsilon\right\}\right)=0 \\
& \Longleftrightarrow \forall \varepsilon>0 ; \forall \delta>0, \exists n_{0}(\delta) \forall n \in \mathbb{N}, n \geq n_{0}(\delta) \\
& \Longrightarrow \mu\left(\left\{t \in X:\left|f_{n}(t)-f(t)\right| \geq \varepsilon\right\}\right)=0 .
\end{aligned}
$$

Thus for all $k \in \mathbb{N}^{*}$, we infer $\varepsilon=\delta=2^{-k}$ that will give for all $k \in \mathbb{N}^{*}$, there exists $n_{k}=n_{0}\left(2^{-k}\right) \in \mathbb{N}$. Then for all $n \in \mathbb{N}, n \geq n_{k}$ implies that $\mu\left(\left\{t \in X:\left|f_{n}(t)-f(t)\right| \geq\right.\right.$ $\left.\left.2^{-k}\right\}\right)<2^{-k}$.

We will choose the $n_{k}$ as an increasing sequence so that $n_{1}<n_{2}<\cdots<n_{k+1}$. We infer

$$
E_{k}=\left\{t \in X:\left|f_{n_{k}}(t)-f(t)\right| \geq 2^{-k}\right\}
$$

It is clear that $\mu\left(E_{k}\right)<2^{-k}$. Take $E=\bigcap_{\partial=1}^{\infty}\left(\bigcup_{k \geq \partial} E_{k}\right)$.

So

$$
\mu\left(\bigcup_{k \geq \partial} E_{k}\right)=\oplus_{k \geq \partial} \mu\left(E_{k}\right)<\oplus_{k \geq \partial} 2^{-k}=g^{-1}\left(\sum_{k \geq \partial} g\left(2^{-k}\right)\right) \leq 2^{-\partial+1}=2 \cdot 2^{-\partial} .
$$

But for all $\partial \in \mathbb{N} ; E \subset \bigcup_{k \geq \partial} E_{k}$, so $\mu(E) \leq \mu\left(\bigcup_{k \geq \partial} E_{k}\right)<2.2^{-\partial}$ implies that $\mu(E)=0$.

If $t \notin E$ if and only if $t \notin \bigcap_{\partial \geq 1}\left(\bigcup_{k \geq \partial} E_{k}\right)$ if and only if for all $\partial \in \mathbb{N}, t \notin \bigcup_{k \geq \partial} E_{k}$ if and only if for all $\partial \in \mathbb{N}$, for all $k \in \mathbb{N}, k \geq \partial$ implies that $t \notin E_{k}$ if and only if for all $\partial \in \mathbb{N}$, for all $k \in \mathbb{N}, k \geq \partial$ implies that $\left|f_{n_{k}}(t)-f(t)\right|<2^{-k}$.

When $k \rightarrow \infty$ then $\left|f_{n_{k}}(t)-f(t)\right| \rightarrow 0$ that is to say $\lim _{k \rightarrow \infty} f_{n_{k}}(t)=f(t)$. Thus $f_{n_{k}}$ converges outside the set $E$. In other words $f_{n_{k}} \rightarrow f \mu$ a.e.

Proposition 2.13. Let $\mu$ be a $\sigma$ - $\oplus$-decomposable measure and $f$ an extended realvalued measurable function pseudo-integrable such that for all $A \in \Omega, \int_{A} f \otimes \mu=0$, then $f=0 \mu$ a.e.

Proof. We infer that $E=\{t \in X: f(t) \neq 0\}$. Let

$$
E_{n}=\left\{t \in X: f(t)>\frac{1}{n}\right\}, \quad F_{n}=\left\{t \in X: f(t)<-\frac{1}{n}\right\} .
$$

It is clear that

$$
E=\bigcup_{n=1}^{\infty}\left(\left\{t \in X: f(t)>\frac{1}{n}\right\} \cup\left\{t \in X: f(t)<-\frac{1}{n}\right\}\right) .
$$

But we know that $f$ is measurable then for all $n \in \mathbb{N}^{*}, E_{n} \in \Omega, F_{n} \in \Omega$.

By using the hypothesis of the proposition we obtain

$$
\begin{aligned}
0 & =\int_{\Omega} f \otimes \mu \geq \int_{E_{n}} f \otimes \mu \geq \int_{E_{n}} \frac{1}{n} \otimes \mu \geq 0 \\
& \Leftrightarrow \int_{E_{n}} \frac{1}{n} \otimes \mu \leq 0 \\
& \Longleftrightarrow g^{-1}\left(\int_{E_{n}} g \circ\left(\frac{1}{n}\right) d \gamma\right) \leq 0
\end{aligned}
$$




$$
\begin{aligned}
& \Leftrightarrow \int_{E_{n}} g\left(\frac{1}{n}\right) d \gamma \leq g(0)=0 \\
& \Leftrightarrow g\left(\frac{1}{n}\right) \gamma\left(E_{n}\right)=0 \\
& \Leftrightarrow \gamma\left(E_{n}\right)=0 \quad \forall n \in \mathbb{N}^{*} .
\end{aligned}
$$

Or $g \circ \mu=\gamma$ so for all $n \in \mathbb{N}, g \circ \mu\left(E_{n}\right)=\gamma\left(E_{n}\right)=0$ implies that $\mu\left(E_{n}\right)=0$ for all $n \in \mathbb{N}^{*}$. We also have

$$
\begin{aligned}
\forall n \geq 1, \quad 0 & =\int_{F_{n}} f \otimes \mu \leq \int_{F_{n}}-\frac{1}{n} \otimes \mu \\
& =-\int_{F_{n}} \frac{1}{n} \otimes \mu \\
& \Leftrightarrow g^{-1}\left(\int_{F_{n}} g\left(\frac{1}{n}\right) d \gamma\right) \leq 0 \\
& \Longleftrightarrow \gamma\left(F_{n}\right)=0 .
\end{aligned}
$$

Thus for all $n \geq 1, \mu\left(F_{n}\right)=0$.

But

$$
E=\bigcup_{n=1}^{\infty}\left(\left\{t \in X: f(t)>\frac{1}{n}\right\} \cup\left\{t \in X: f(t)<-\frac{1}{n}\right\}\right)=\bigcup_{n=1}^{\infty}\left(E_{n} \bigcup F_{n}\right) .
$$

Then

$$
\begin{aligned}
\mu(E) & =\mu\left(\bigcup_{n=1}^{\infty}\left(E_{n} \bigcup F_{n}\right)\right) \\
& =\mu\left[\left(E_{1} \cup F_{1}\right) \cup\left(E_{2} \cup F_{2}\right) \cup \cdots\right] \\
& =\mu\left(E_{1}\right) \oplus \mu\left(F_{1}\right) \oplus \mu\left(E_{2}\right) \oplus \mu\left(F_{2}\right) \oplus \cdots=0 .
\end{aligned}
$$

That means $f=0 \mu$ a.e.

\section{Fuzzy measurable function}

THEOREM 3.1. Let $(X, \Omega, \mu)$ be a finite pseudo-measure space, the following hold for any $f, h, k \in L^{1}(\mu)$

(1) $(f /(1+|f|))$ is pseudo-integrable.

(2) If we infer $\sigma(f, h)=\int_{X}(|f-h| /(1+|f-h|)) \otimes \mu$

(a) $\sigma(f, h)=0$ if and only if $f=h \mu$ a.e.,

(b) $\sigma(f, h)=\sigma(h, f)$,

(c) $\sigma(f, k) \leq \sigma(f, h) \oplus \sigma(h, k)$.

Proof. (1) We have

$$
\begin{aligned}
\forall f \in L^{1}(\mu), \quad 0 \leq \frac{|f|}{1+|f|}<1 \\
\Leftrightarrow 0 \leq \int_{X} \frac{|f|}{1+|f|} \otimes \mu<\int_{X} 1 \otimes \mu \\
\Leftrightarrow 0 \leq \int_{X} \frac{|f|}{1+|f|} \otimes \mu<\mu(X)<\infty .
\end{aligned}
$$


Thus $|f| /(1+|f|)$ is pseudo-integrable so $f /(1+|f|)$ is also pseudo-integrable.

(2) (a) $\sigma(f, h)=0$ if and only if $f=h \mu$ a.e.

$(\Rightarrow)$ Suppose that $\sigma(f, h)=0$ then $\int_{X}(|f-h| /(1+|f-h|)) \otimes \mu=0$. We infer that

$$
E=\{t \in X:|f(t)-h(t)|>0\}, \quad E_{n}=\left\{t \in X:|f(t)-h(t)|>\frac{1}{n}\right\} .
$$

It is clear that $E=\bigcup_{n=1}^{\infty} E_{n}$. In fact if $t \in E$ then the absolute value of $|f(t)-h(t)|>0$ that imply there exists $n_{0} \in \mathbb{N}^{*}$ such that

$$
|f(t)-h(t)|>\frac{1}{n_{0}} \Longrightarrow t \in \bigcup_{n=1}^{\infty}\left\{t \in X:|f(t)-h(t)|>\frac{1}{n}\right\} \Rightarrow E \subset \bigcup_{n=1}^{\infty} E_{n} .
$$

Conversely, if $t \in \bigcup_{n=1}^{\infty}\{t \in X:|f(t)-h(t)|>1 / n\}$, then there exists $n_{1} \in \mathbb{N}^{*}$ such that absolute value $|f(t)-h(t)|>1 / n_{1}$ imply absolute value of $|f(t)-h(t)|>0$ that means $t \in E$. So $t \in \bigcup_{n \geq 1} E_{n} \subset E$.

We infer

$$
\varphi: \mathbb{R}^{+} \rightarrow \mathbb{R}^{+}, \quad t \rightarrow \frac{t}{(1+t)}
$$

we obtain $\varphi^{\prime}(t)=1 /(1+t)^{2}$ thus $\varphi$ is increasing. Let $t \in E_{n}$, then

$$
\frac{|f(t)-h(t)|}{1+|f(t)-h(t)|} \geq \frac{1 / n}{1+1 / n}=\frac{1}{n+1} .
$$

By using the hypothesis $\sigma(h, f)=0$ we obtain

$$
\begin{aligned}
0 & =\int_{X} \frac{|f-h|}{1+|f-h|} \otimes \mu \geq \int_{E_{n}} \frac{|f-h|}{1+|f-h|} \otimes \mu \geq \int_{E_{n}} \frac{1}{n+1} \otimes \mu \geq 0 \\
& \Longleftrightarrow 0=\frac{1}{n+1} \otimes \mu\left(E_{n}\right) \quad \forall n \in \mathbb{N} \text { that is to say } \mu\left(E_{n}\right)=0 \quad \forall n \in \mathbb{N} .
\end{aligned}
$$

Since $\mu(E)=\mu\left(\bigcup_{n=1}^{\infty} E_{n}\right)=\oplus_{n=1}^{\infty} \mu\left(E_{n}\right)=\mu\left(E_{1}\right) \oplus \mu\left(E_{2}\right) \oplus \cdots=0$, then $\mu(E)=0$ that is to say $f=h \mu$ a.e.

$(\Leftarrow)$ By hypothesis $f=h \mu$ a.e. Then $\mu(E)=0$.

Thus

$$
\int_{X} \frac{|f-h|}{1+|f-h|} \otimes \mu=\int_{E} \frac{|f-h|}{1+|f-h|} \otimes \mu=0 .
$$

(b) $\sigma(f, h)=\sigma(h, f)$ is obvious.

(c) For all $f, h, k \in L^{1}(\mu)$ we $|f-h| \leq|f-k|+|k-h|$.

So

$$
\begin{aligned}
\frac{|f-h|}{1+|f-h|} & \leq \frac{|f-k|+|k-h|}{1+|f-k|+|k-h|} \\
& =\frac{|f-k|}{1+|f-k|+|k-h|}+\frac{|k-h|}{1+|f-k|+|k-h|} \\
& \leq \frac{|f-k|}{1+|f-k|}+\frac{|k-h|}{1+|k-h|} .
\end{aligned}
$$


And we obtain by using the properties of pseudo-additive integral in [4]

$$
\begin{aligned}
\int_{X} \frac{|f-h|}{1+|f-h|} \otimes \mu & \leq \int_{X}\left[\frac{|f-k|}{1+|f-k|}+\frac{|k-h|}{1+|k-h|}\right] \otimes \mu \\
& =\int_{X} \frac{|f-k|}{1+|f-k|} \otimes \mu \oplus \int_{X} \frac{|k-h|}{1+|k-h|} \otimes \mu \Longleftrightarrow \sigma(f, h) \\
& \leq \sigma(f, k) \oplus \sigma(k, h) .
\end{aligned}
$$

REMARK 3.2. Theorem 3.1 implies that the space $(S(\mu), \sigma(\cdot, \cdot))$ of fuzzy measurable function is a pseudo-metric space.

4. Application of this extension. Let $(X, \Omega, \mu)$ be a pseudo-measure space and $Y$ be a Banach space on $(-\infty, \infty)$.

DEFINITION 4.1. A vector-valued function $f: X \rightarrow Y$ will be called pseudo-Pettis weakly integrable if $x^{*} f$ is pseudo-integrable for each $x^{*} \in Y^{*}$ (dual of $Y$ ). Moreover, if for every $A \in \Omega$ there exists $x_{A} \in Y$ such that

$$
x^{*}\left(x_{A}\right)=\int_{A} x^{*}(f) \otimes \mu \quad \forall x^{*} \in Y^{*},
$$

then $f$ is called pseudo-Pettis integrable and $x_{A}$ is said to be the pseudo-Pettis integral of $f$ over $A$, denoted by

$$
x_{A}=(P) \int_{A} f \otimes \mu
$$

and we have $x^{*}\left((P) \int_{A} f \otimes \mu\right)=\int_{A} x^{*}(f) \otimes \mu$ and then

$$
\int_{A} x^{*}(f) \otimes \mu=\int_{A}\left(x^{*}(f)\right)^{+} \otimes \mu-\int_{A}\left(x^{*}(f)\right)^{-} \otimes \mu .
$$

The pseudo-Pettis integral built above is well defined because $f$ is a vector-valued function defined on $X$ and the numerical function $x^{*} f$ is pseudo-integrable according to Definition 2.9.

REMARK 4.2. If $Y=(-\infty, \infty)$ then the pseudo-Pettis integral and pseudo integral are the same.

Conclusion. The extension of the concept of fuzzy integral based on pseudoadditive integral is very important, it will allow us to introduce Pettis integral and Bochner integral in the theory of fuzzy integral. Those concepts will be deeply studied in a subsequent paper.

\section{REFERENCES}

[1] P. R. Halmos, Measure Theory, D. Van Nostrand, New York, 1950. MR 11,504d. Zbl 0040.16802 .

[2] H. Ichihashi, H. Tanaka, and K. Asai, Fuzzy integrals based on pseudo-additions and multiplications, J. Math. Anal. Appl. 130 (1988), no. 2, 354-364. MR 89m:28039. Zbl 0656.28013. 
[3] E. P. Klement and S. Weber, Generalized measures, Fuzzy Sets and Systems 40 (1991), no. 2, 375-394. MR 92a:28015. Zbl 0733.28012.

[4] T. Mamadou, The fuzzy integral based on pseudo-additive measure and the convergence theorem, J. Math. (PRC) 21 (2001), 403-408.

[5] T. Murofushi and M. Sugeno, Fuzzy t-conorm integral with respect to fuzzy measures: generalization of Sugeno integral and Choquet integral, Fuzzy Sets and Systems 42 (1991), no. 1, 57-71. MR 92m:28026. Zbl 0733.28014.

[6] E. Pap, Decomposable measures and nonlinear equations, Fuzzy Sets and Systems 92 (1997), no. 2, 205-221. MR 98m:28048.

[7] M. Sugeno, Theory of fuzzy integral and its application, Ph.D. thesis, Tokyo Institute of Technology, Tokyo, 1974.

[8] M. Sugeno and T. Murofushi, Pseudo-addition measure and integrals, J. Math. Appl. 122 (1987), 197-222.

[9] S. Weber, $\perp$-decomposable measures and integrals for Archimedean t-conorms $\perp$, J. Math. Anal. Appl. 101 (1984), no. 1, 114-138. MR 86c:26015. Zbl 0614.28019.

[10] L. A. Zadeh, Fuzzy sets, Information and Control 8 (1965), 338-353.

Mamadou Traore: Department of Mathematics, Harbin Institute of Technology, HARBIN 150001, CHINA

E-mail address: mamadoutr@yahoo.com 


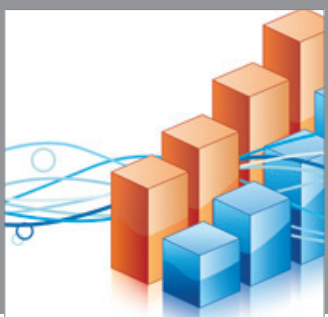

Advances in

Operations Research

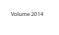

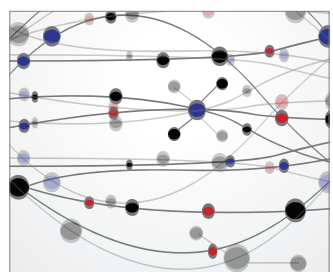

\section{The Scientific} World Journal
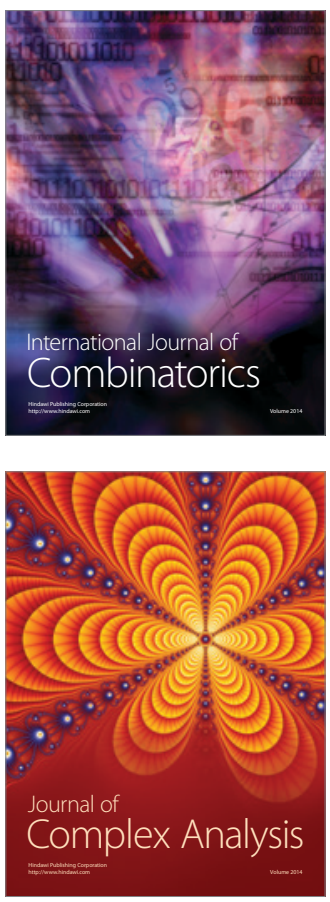

International Journal of

Mathematics and

Mathematical

Sciences
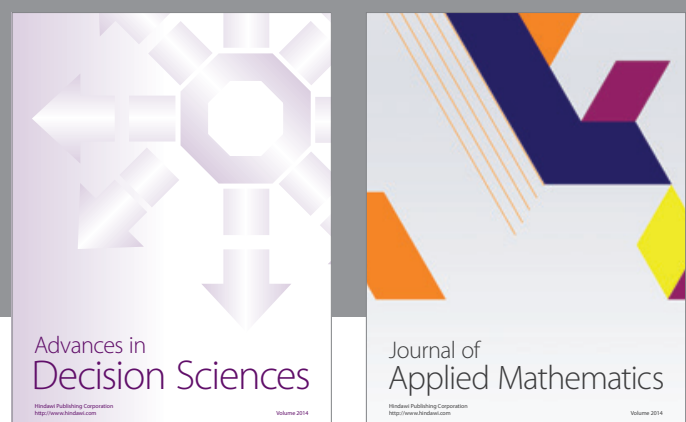

Journal of

Applied Mathematics
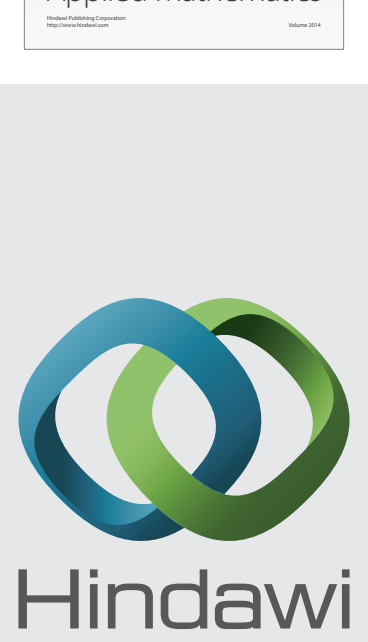

Submit your manuscripts at http://www.hindawi.com
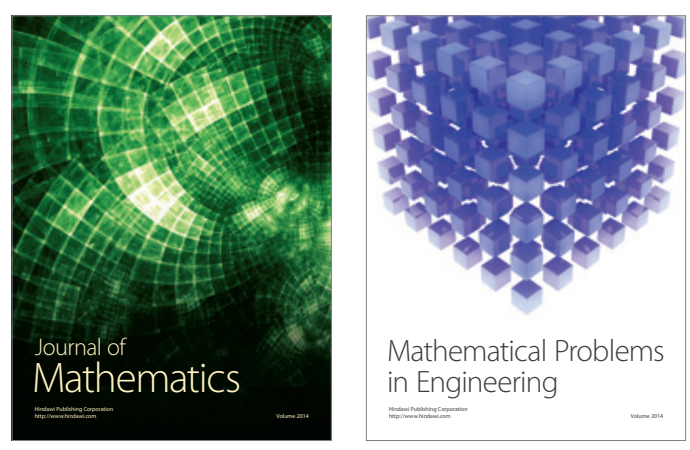

Mathematical Problems in Engineering
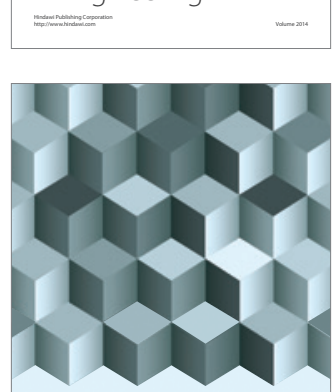

Journal of

Function Spaces
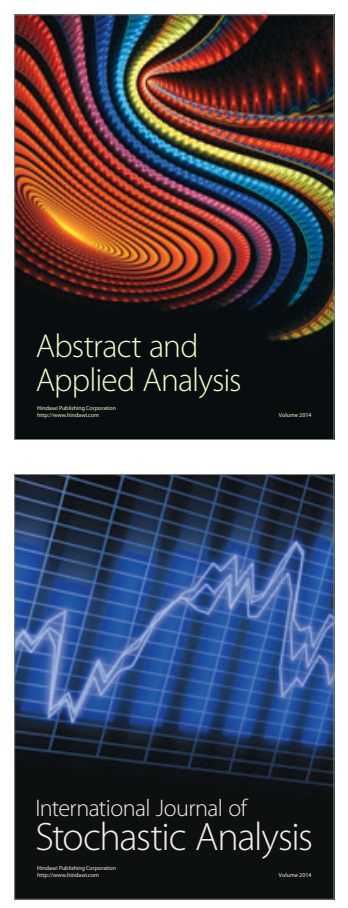

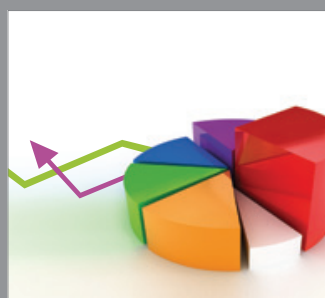

ournal of

Probability and Statistics

Promensencen
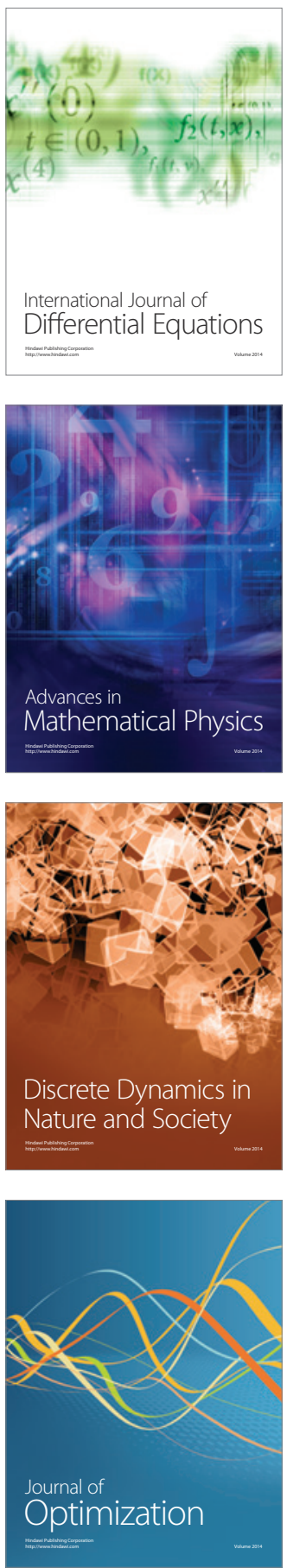